\title{
Paediatric dentistry training for Yorkshire and Humber
}

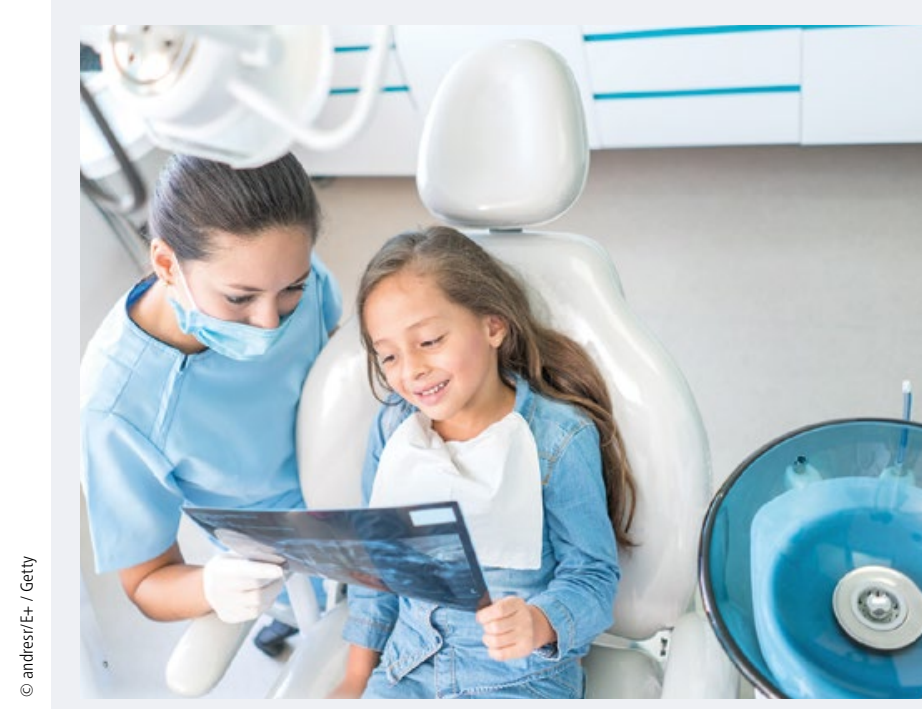

The selection process takes place in Leeds this September for 12 dentists who will be allocated places on a new paediatric dentistry training programme in Yorkshire and Humber. Interest in the level 2 complexity care training funded by Health Education England, Yorkshire and Humber has surpassed the expectations of the organisers who were inundated with expressions of interest within days of going public.

The level 2 dentist was defined in the Commissioning Standard for Paediatric Dentistry published in 2016. Qualified dentists are trained to provide an enhanced level of care, managing more complex conditions, such as dental anomalies, traumatic dental injuries and managing children who are anxious or phobic.

The 24-month programme is due to start in January, lasts for two years and will equip the participants for level 2 accreditation in paediatric dentistry. It is being led by Rachael Nichol, a Consultant in Paediatric Dentistry in Wakefield supported by Esther McDerra, a Specialist in Paediatric Dentistry based in Dewsbury. They have the backing of key dentists in Yorkshire and Humber, including James Spencer, Postgraduate Dental Dean, Stephen Fayle, the Chairman of the Managed Clinical Network in Paediatric Dentistry and Simon Hearnshaw, of Health Education England and Chairman of the North Yorkshire and Humber Local Dental Network.

Regional commissioners have given their blessing to the programme. Discussions are now underway over remuneration in the hope that Units of Dental Activity (UDAs) can be replaced with a sessional rate to reflect the time required for management of young patients or patients with special educational needs.

Rachael has a record of innovating. As a trainee, she was the first in Yorkshire to have paediatric dentistry specialist training which was split between the Community Dental Service and Hospital, in Doncaster and Leeds Dental Institute, respectively. When Rachael arrived in Wakefield in 2019, she was the first Consultant in Paediatric Dentistry to be appointed to the Mid Yorkshire Hospitals Trust.

Rachael is now looking forward to getting the training programme established: 'I cannot believe the amount of interest there has been. We still need more consultants and specialists but having level 2 dentists in the community and in general practice will take the pressure off secondary care services and allow more children to be treated more quickly and closer to home'.

Stephen Fayle, a British Society of Paediatric Dentistry (BSPD) spokesman, said: 'This is a fantastic step forward. The idea of giving our colleagues in the community and in general practice additional specialty-specific training has been around for some time. It was first proposed in 2004 and successfully trialled in Yorkshire but discontinued after the introduction of the GDS contract of 2006. It's very exciting to see this opportunity to improve dental services for children finally coming to fruition'.

\section{BAOMS and HEE consent e-FACE module launched online}

A new e-learning module on consent has gone live as part of the British Association of Oral and Maxillofacial Surgeons (BAOMS) and Health Education England e-Learning for Healthcare (HEE e-LfH) e-FACE project. This 12th module in the programme is designed to tackle the limited consent knowledge many young trainees have.

The sessions have been developed to demonstrate best practice, and are aimed at dental core trainees (DCTs) and specialty registrars in oral and maxillofacial surgery (OMFS).

Loz Newman, the consultant maxillofacial/head and neck surgeon who conceived this key module, said that consent is always important to surgeons: 'This is increasingly so in light of the 2015 Montgomery V Lanarkshire Health Board case' (https://bit. ly/2GYgGHs).

He explained that the module came about following a talk he gave at the Royal College of Surgeons of England (RCSEng): 'We were all concerned that young trainees are often sent to seek consent from patients inappropriately, for procedures where they have limited knowledge. We felt that not infrequently they were ill prepared for the task. Consent is barely taught in the undergraduate curriculum, and there is little in the postgraduate curriculum'

The idea is to provide consent process training for DCTs that offers them some form of protection with a certificate awarded once the module is complete. This shows that they have had some formal training in consent that relates to specific surgical interventions in addition to aiding their learning.

'We thought it would be sensible to write clinical scenarios that would be more relevant and easier to digest than the more dry, legalese textbook approach to consent,' said Dr Newman.

DCTs currently working in OMFS departments should already be aware of the free-to-use material on the eLfH website. This has a range of easy-to-access e-learning across all of medicine and dentistry.

For further information on the e-FACE e-learning programme visit: https://www.e-lfh.org.uk/programmes/ oral-and-maxillofacial-surgery/. 